\title{
Efecto de un programa basado en entrenamiento funcional sobre la capacidad de velocidad de practicantes de patinaje de entre 9 y 11 años de edad ${ }^{*}$
}

\author{
Rubiel Antonio Barrera Izquierdo* \\ Jhon F. Ramírez-Villada**
}

Recibido: marzo 13 de 2018 • Aceptado: junio 7 del 2018

\section{Resumen}

Este trabajo planteó determinar los efectos de un programa de entrenamiento funcional sobre la velocidad de carrera de deportistas de patinaje de entre 9 y 11 años. 30 jóvenes atletas fueron asignados aleatoriamente a un grupo de entrenamiento basado en movimientos naturales centrados en la fuerza GE $(n=15)$ y un grupo control GC $(n=15)$. El GE participó en un programa basado en entrenamiento funcional durante 12 semanas, con una frecuencia de 3 días, y una duración por sesión de 2 horas. Fueron registradas las variables morfológicas (peso, talla, índice de masa corporal, IMC), de fuerza

" Artículo de investigación, con financiación propia. Citar como: Barrera Izquierdo, R. A. y Ramírez-Villada, J. F. (2018). Efecto de un programa basado en entrenamiento funcional sobre la capacidad de velocidad de practicantes de patinaje de entre 9 y 11 años de edad. Cuerpo, Cultura y Movimiento, 8(2), 13-34 DOI: https://doi.org/10.15332/2422474X.5124

"* Magíster en Ciencias y Tecnologías del Deporte y la Actividad Física. Universidad de los Llanos, Villavicencio, Meta, Colombia. Correo electrónico: ruanbaiz1007@gmail.com

"**Posdoctor en Narrativa y Ciencia, doctor en Ciencias Aplicadas a la Actividad Física y el Deporte, docente e investigador asociado, Universidad de Antioquia, Medellín, Colombia. Correo electrónico: jhon.ramirez3@udea.edu.co 
explosiva (tiempo de vuelo, altura, velocidad de despegue y potencia), velocidad dinámica y estática (200 metros y 50 metros) en dos momentos diferentes (pre y pos test). No se registraron diferencias significativas $(\mathrm{p} \leq 0.05 \mathrm{y}$ 0.01 ) en las variables morfológicas, excepto para el perímetro de la pierna. Diferencias significativas $(\mathrm{p} \leq 0.05$ y 0.01$)$ fueron registradas para las variables tiempo de vuelo, altura y velocidad en los gestos salto vertical (SJ), salto con contramovimiento $(\mathrm{CMJ})$ y salto con contramovimiento y oscilación de brazos (CMJas), así como para la velocidad dinámica y estática en 50 y 200 metros. Los resultados sugieren que 12 semanas de entrenamiento funcional con movimiento naturales centrados en la fuerza muscular mejoran la expresión de fuerza explosiva e inducen cambios en la condición física (pruebas estática y dinámica) de patinadores.

Palabras clave: entrenamiento funcional, fuerza explosiva, velocidad, patinaje de carreras. 


\section{Effects of functional training program on velocity performance of skating's athletes between 9 and 11 years}

Abstract

This work aimed to determine the effects of a functional training program on the running speed of skaters from 9 to 11 years old. 30 young athletes were randomly assigned to a training group based on natural movements centered on the GE force $(n=15)$ and a GC control group $(n=15)$. The GE participated in a program based on functional training for 12 weeks, with a frequency of 3 days, and a duration per session of 2 hours. The morphological variables (weight, height, body mass index, BMI), explosive force (flight time, height, take-off speed and power), dynamic and static speed (200 meters and 50 meters) were recorded at two different times (pre and post test). There were no significant differences ( $p \leq 0.05$ and 0.01$)$ in the morphological variables, except for the perimeter of the leg. Significant differences ( $\mathrm{p} \leq 0.05$ and 0.01 ) were recorded for the flight time, height and speed variables in the vertical jump (SJ), countermovement jump (CMJ) and countermovement jump and arm swing (CMJas) gestures, as well as for the dynamic and static speed in 50 and 200 meters. The results suggest that 12 weeks of functional training with natural movement focused on muscle strength improve the expression of explosive force and induce changes in the physical condition (static and dynamic tests) of skaters.

Keywords: functional training, explosive strength, speed, racing skating. 


\section{Introducción}

El patinaje de carreras es una de las modalidades más destacadas a nivel competitivo mundial, tanto por la exigencia física y mental a la cual se ven expuestos los deportistas, como por la respuesta metabólica que se requiere en las diferentes pruebas competitivas (Zapata, Vicente y Rábago, 2006).

No obstante, la especialización temprana amenaza la salud e integridad de sus practicantes debido a que los modelos de programación, las variables y los contenidos son en la mayoría de los casos, procesos copiados del trabajo realizado con jóvenes o adultos. Por lo tanto, es necesario identificar nuevas estrategias metodológicas que permitan mejorar el rendimiento físico, técnico y táctico en edades tempranas, sin exponer a los sujetos a cargas o contenidos que generen lesión, discapacidad o abandono de la práctica deportiva $(\mathrm{Na}-$ clerio, 2011).

Al respecto, algunos estudios han descrito la importancia de orientar estímulos para el mejoramiento de los procesos del sistema nervioso central y periférico (patrones de coordinación intra e intermuscular (Ozmun, Mikesky y Surburg, 1994; Blimkie et al., 1989) así como para el sistema osteomuscular de los deportistas en general. En el caso específico de infantes, los datos revelan que las ganancias en la respuesta funcional (capacidades condicionales) son, en su mayoría, debidas a las adaptaciones neurológicas y neuromotoras (Sailors y Berg, 1987), más que a procesos de hipertrofia muscular.

Otros estudios reportan cambios positivos en las expresiones de tensión (isocinética e isotónica), así como en la actividad electromiográfica de los músculos involucrados en el gesto técnico de practicantes de patinaje de carreras (Ozmun, Mikeski y Surburg, 1994), sin que sean significativos los cambios de la masa muscular, datos reveladores de cara a orientar los contenidos propios de una sesión de entrenamiento en edades tempranas.

Entre los modelos de programación del entrenamiento aplicados en infantes, algunos estudios sugieren analizar nuevas formas de orientar sesiones de entrenamiento de fuerza por sus efectos sobre los patrones de coordinación intra e intermuscular que inciden positivamente en la calidad de los gestos técnicos, siempre y cuando se controlen los contenidos, variables de programación (la intensidad, la duración de los ejercicios o sus contenidos, entre otros), frecuencia y duración total del proceso (American Academy of 
Pediatrics, 2000; American College of Sports Medicine, 1993; Faigenbaum, Zaichkowsky, Westcott, Micheli y Fehlandt, 1993).

Por lo tanto, es necesario profundizar sobre nuevos modelos, variables y contenidos, que, respetando los límites fisiológicos y morfológicos propios de la edad, así como su etapa de maduración y desarrollo, permitan elevar el rendimiento técnico y táctico de los niños, preparándolo para niveles más especializados en edades posteriores, sin exponer su salud y calidad de vida.

Atendiendo a lo expuesto, en la presente investigación se planteó como objetivo el determinar los efectos que genera un programa de fuerza basado en entrenamiento funcional sobre la capacidad de velocidad en patinadores de 9 y 11 años, no solo por los posibles aportes que brinda a la preparación física en estas edades, sino también por la esencia lúdica y jugada que ofrece el modelo basado en entrenamiento funcional.

\section{Materiales y métodos}

Se realizó un estudio de diseño cuasiexperimental de dos grupos (grupo experimental "GE" y grupo control "GC") con preprueba y posprueba. Entre los criterios de inclusión fueron considerados: la edad (9 a 11 años), el género (masculino/femenino), la experiencia en la práctica de la modalidad deportiva activa (1 año mínimo), la certificación clínica para la práctica otorgada por el equipo biomédico, la autorización por parte de los padres de familia y el deportista para hacer parte del estudio.

Los infantes fueron excluidos del estudio si presentaban problemas osteomusculares limitantes o que pudieran verse condicionados por los estímulos a aplicar en el estudio, alteración de la columna vertebral, de miembros superiores o inferiores, amputaciones, secuelas de fracturas, prótesis, tratamiento de enfermedades cardiovasculares y afecciones articulares, así como haber tenido alguna lesión limitante 6 meses antes de participar en el programa de entrenamiento funcional.

Un total de 30 sujetos fueron asignados aleatoriamente al GE $(n=15)$ y al GC $(n=15)$. Al final del proceso fueron excluidos cuatro participantes debido a la asistencia irregular a los entrenamientos, los controles o por el abandono total del programa $(\mathrm{GE}=12 / \mathrm{GC}=14)$. 
El estudio fue ejecutado siguiendo los parámetros establecidos en la declaración de Helsinki (2014) de la Asociación Médica Mundial. De igual manera, fue sometido a valoración y aprobación por parte del comité bioético de las instituciones figurantes las cuales otorgaron número de aprobación bioético con código 0820082014.

Por lo tanto, siguiendo las recomendaciones bioéticas, los responsables de los menores de edad legal, las instituciones participantes y los profesionales a cargo, aprobaron y firmaron el consentimiento, asentimiento y los oficios de autorización respectivos.

\section{Técnica de muestreo}

Para el estudio, la selección de la muestra es no probabilística, lo que implicó que la selección de las unidades de análisis no dependiera de la probabilidad sino de las causas relacionadas con las características de la investigación obedeciendo a los criterios de inclusión fijados, aunque la conformación de los grupos fue aleatoria, es decir, cualquiera de los individuos tenía la misma posibilidad de pertenecer al GE o al GC.

\section{Descripción de los procedimientos, técnicas e instrumentos}

Antes de llevar a cabo el trabajo de campo, se procedió a realizar una prueba de confiabilidad instrumental y procedimental (error intraobservador e interobservador) con diez individuos deportistas diferentes a los incluidos en el estudio, la cual arrojó resultados con error estimado inferior al $2 \%$ (confiabilidad superior al $95 \%$ ).

\section{Valoración antropométrica}

Se realizó la valoración antropométrica básica de la variable peso, con una báscula digital Omron BF511, con precisión de $0.0 \mathrm{~kg}$ a $40.0 \mathrm{~kg}: \pm 0.4 \mathrm{~kg}$, la variable talla con un tallímetro marca SECA $®$, y los perímetros (cintura, cadera, muslo y pierna) con una cinta métrica flexible e inextensible marca 
Stanley®. La ejecución de las mediciones y el control de las fuentes de error siguieron las recomendaciones establecidas en el manual de cineantropometría (Quintana, 2005), que están basadas en la Sociedad Internacional para el Avance de la Cineantropometría (Marfell-Jones, 2001).

\section{Valoración funcional}

\section{Pruebas para la valoración de la fuerza generada por miembros inferiores}

Para el conocimiento de la fuerza generada por los miembros inferiores se empleó ergometría isoinercial (Romero et al., 2014) con el uso de distintos tipos de salto y variando el ángulo articular. La fuerza explosiva se midió por la respuesta del sujeto a un salto máximo vertical (Bosco, 1994) (Squatjump [SJ]: partiendo desde una posición de flexión de la rodilla de $90^{\circ}$, con manos en la cintura, se salta elevando el centro de gravedad), un salto máximo con contramovimiento (Countermovement Jump [CMJ]: de pie, con extensión completa de las rodillas y las manos en la cintura, se baja a $90^{\circ}$ y se salta) y un salto máximo con contramovimiento y coordinación de los miembros superiores (Countermovement Jump arm swing [CMJas]: similar al anterior, pero con la colaboración de los miembros superiores para lograr una mayor altura). Se ejecutaron de 3 a 5 saltos por cada prueba (SJ, CMJ y CMJas) y se midieron la altura, el tiempo de vuelo y la velocidad más representativas para su posterior análisis. En la medición de la fuerza explosiva se utilizó una alfombra de saltos AXOM JUMP Technology®.

\section{Pruebas de velocidad}

Las pruebas de velocidad-desplazamiento dinámica y estática en pista en los 50 y 200 metros, se realizó mediante el protocolo establecido por la Federación Internacional de Patinaje (Federatión International de Roller Sport -FIRS, 1990), los cuales son descritos de la siguiente manera: 
Prueba de 200 metros estáticos-contrarreloj: se disputa en circuito, el corredor ha de tomar la salida situándose entre dos líneas separadas $50 \mathrm{~cm}$ entre sí, y mediante una técnica de salida especial para este tipo de pruebas, arrancará desde parado hasta la línea de meta, en el menor tiempo posible.

Prueba 200 metros lanzados: se disputa en pista, el corredor dispone de aproximadamente 150 metros para salir y ganar velocidad, de manera que, al pasar por la línea de salida, dispute una vuelta en el menor tiempo posible (clasificación por tiempos con un único intento).

Prueba de los 50 metros: se tuvo en cuenta el protocolo de 200 metros estáticos contrarreloj donde solo se varió la distancia recorrida para la toma de los tiempos.

En todas las pruebas fueron empleados cronómetros marca Casio HS80 TW-1EF, con una ubicación cada 50 metros hasta cubrir la totalidad del recorrido.

\section{Programa de entrenamiento funcional}

El programa de entrenamiento funcional se desarrolló durante 12 semanas, con una frecuencia de 3 veces por semana y una duración de 2 horas por sesión de trabajo, donde se establecieron unos niveles de trabajo de multisaltos con desarrollo multidireccional (adelante, atrás, lados, diagonales), ejercicios unipodales, ejercicios de fortalecimiento de la zona media, trabajo con lastres y ejercicios propios de la disciplina del patinaje, atendiendo a los principios del acondicionamiento físico general y del entrenamiento funcional sugeridos por diversos autores (Platonov, 2001; García, 1999; Bompa, 2003; Ravé, Valdivielso y Gaspar, 2007; Aón, 2011; Aón, Birrum y Lafranco, 2001; Heredia, Chulvi, Donate, Soro y Costa, 2007; Chulvi, 2008; Heredia, Elvar y Peña, 2011), con una distribución porcentual y dosificación de la carga para el período de entrenamiento (tabla 1). 
Tabla 1. Distribución porcentual y dosificación de la carga para el período de entrenamiento

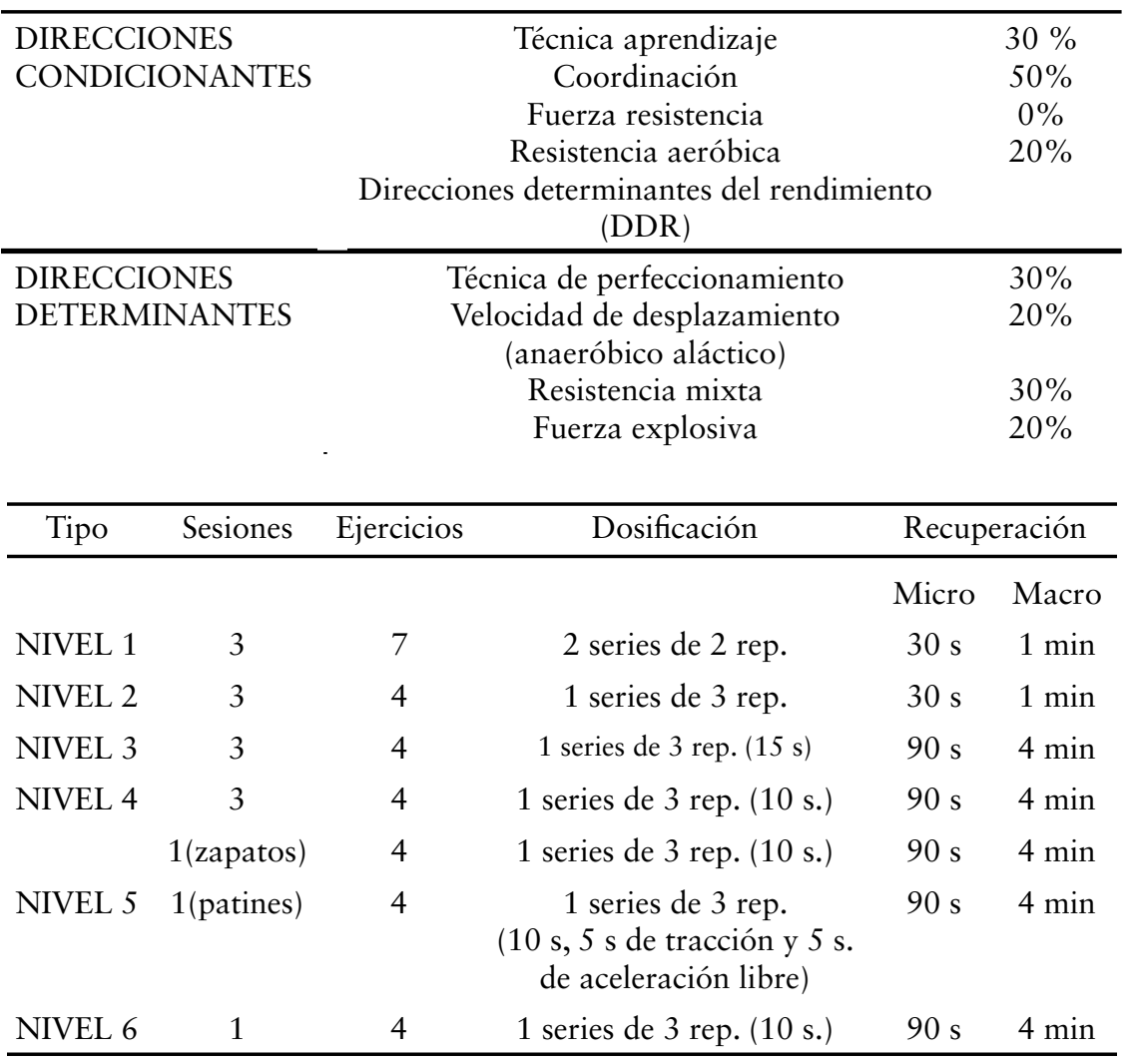

Fuente: elaboración propia.

\section{Análisis estadístico}

Fue aplicada la prueba de bondad o ajuste de normalidad a través de la prueba de Shapiro-Wilk y distribución gráfica con curva de normalidad. Se establecieron niveles de diferencia del $5 \%$ con un intervalo de confianza del $95 \%$ y se consideraron estadísticamente significativos los valores de $\mathrm{p}<0.05$. Considerando el tamaño muestral, las pruebas de normalidad y la homocedasticidad, fueron seleccionadas las pruebas no paramétricas de 
Wilcoxon (muestras relacionadas), de Krus Kal-Wallis (muestras independientes) y de Sperman (correlaciones). El análisis estadístico fue realizado empleando el paquete estadístico SSPS versión 22.

\section{Resultados y discusión}

\section{Variables morfológicas}

En la Tabla 2 se presentan las características de partida para el GE y el GC respectivamente, donde se puede apreciar que no existen diferencias significativas entre los grupos. Además, se describen los cambios en las variables peso, IMC, perímetro de muslo y perímetro de cadera, tanto en el GC como en el GE, tras 12 semanas de participación en un programa de entrenamiento funcional. Al finar de la intervención, se observan diferencias significativas $(\mathrm{p} \leq 0.05$ y 0.01$)$ en el perímetro de pierna en el GE, que corresponde a $9.1 \%(9.10 \pm 1.57)$, en tanto el GC no presentó variaciones significativas $(0.76 \pm 3.31 \%)$. 
Tabla 2. Características físicas del grupo experimental (GE) y del grupo control (GC) antes y después de la participación en un programa de entrenamiento funcional

\begin{tabular}{|c|c|c|c|c|c|c|c|}
\hline \multirow[t]{2}{*}{ Variable } & \multicolumn{2}{|c|}{$\mathrm{GE}(\mathrm{n}=12)$} & \multicolumn{2}{|c|}{$\mathrm{GC}(\mathrm{n}=14)$} & \multirow{2}{*}{$\begin{array}{l}\text { GE } \\
\text { Comparación } \\
\text { intramuestral }\end{array}$} & \multirow{2}{*}{$\begin{array}{l}\text { GC } \\
\text { Comparación } \\
\text { intramuestral }\end{array}$} & \multirow{2}{*}{$\begin{array}{l}\text { GE VS GC } \\
\text { Comparación } \\
\text { intermuestral } \\
\text { (Post-test) }\end{array}$} \\
\hline & Pre-test & Post- test & Pre-test & Post- test & & & \\
\hline Peso $(\mathrm{kg})$ & $34.95 \pm 5.22$ & $35.04 \pm 5.19$ & $35.17 \pm 7.37$ & $35.71 \pm 7.41$ & $\mathbb{S}$ & $\mathbb{\$}$ & $\mathbb{S}$ \\
\hline Estatura (m) & $1.4208 \pm 0.08$ & $1.42 \pm 0.08$ & $1.41 \pm 0.09$ & $1.41 \pm 0.09$ & $\$$ & $\mathbb{S}$ & $\$$ \\
\hline $\begin{array}{l}\text { IMC }(\mathrm{kg} / \\
\mathrm{m} 2)\end{array}$ & $17.24 \pm 1.36$ & $17.22 \pm 1.37$ & $17.45 \pm 2.02$ & $17.63 \pm 2.01$ & $\mathbb{S}$ & $\mathbb{S}$ & $\mathbb{S}$ \\
\hline $\begin{array}{l}\text { Perímetro de } \\
\text { muslo }(\mathrm{cm})\end{array}$ & $37.95 \pm 4.93$ & $40.52 \pm 4.93$ & $40.00 \pm 3.98$ & $40.19 \pm 3.99$ & $\mathbb{S}$ & $\mathbb{S}$ & $\$$ \\
\hline $\begin{array}{l}\text { Perímetro de } \\
\text { pierna }(\mathrm{cm})\end{array}$ & $28.26 \pm 1.70$ & $31.09 \pm 1.43$ & $28.76 \pm 3.25$ & $28.98 \pm 3.36$ & $* *$ & $\mathbb{S}$ & $*$ \\
\hline $\begin{array}{l}\text { Perímetro de } \\
\text { cadera }(\mathrm{cm})\end{array}$ & $74.41 \pm 4.80$ & $75.04 \pm 4.56$ & $72.81 \pm 3.30$ & $73.03 \pm 3.19$ & $\mathbb{S}$ & $\mathbb{S}$ & $\$$ \\
\hline
\end{tabular}

Nota: **= valor $\mathrm{p} \leq 0.001 ; \mathbb{\$}=$ No significativo; * valor $\mathrm{p} \leq 0.05$.

Fuente: elaboración propia.

\section{Variables funcionales}

Los cambios en la fuerza explosiva de miembros inferiores fueron analizados a través de indicadores de desempeño (altura de salto, tiempo de vuelo, velocidad de despegue) en SJ, CMJ y CMJas, lo que permitió detectar diferencias significativas $(\mathrm{p} \leq 0.05$ y 0.01$)$ entre el GE y el GC tras 12 semanas de entrenamiento funcional.

En la Tabla 3 se muestran los registros para la altura $(\mathrm{cm})$ en las pruebas de SJ, CMJ y CMJas con diferencias significativas ( $\mathrm{p} \leq 0.05$ y 0.01 ) en favor del GE y con respecto al GC, tras 12 semanas de entrenamiento funcional. La altura $(\mathrm{cm})$ pre/pos varió significativamente en la prueba $\mathrm{SJ}$ en un $12.73 \pm 2.16 \%$ ( $\mathrm{p} \leq 0.05$ y 0.01$)$, CMJ en un $10.02 \pm 2.22 \%$ ( $\mathrm{p} \leq 0.05$ y 0.01 ) y CMJas en un $14.72 \pm 3.72 \%$ ( $\leq \leq 0.05$ y 0.01 ) para el GE. En el caso del GC, los registros de la altura $(\mathrm{cm})$ pre/pos para $\mathrm{SJ}$ fueron de un $5.30 \pm 3.24 \%$, CMJ un $1.71 \pm 1.70 \%$ y CMJas $2.97 \pm 5.01 \%$, sin diferencias significativas encontradas. 
En la Tabla 3 se muestran los registros para el tiempo de vuelo (ms) en las pruebas de SJ, CMJ y CMJas con diferencias significativas $(\mathrm{p} \leq 0.05 \mathrm{y}$ 0.01) en favor del GE y con respecto al GC, tras 12 semanas de entrenamiento funcional. El tiempo de vuelo $(\mathrm{ms})$ pre/pos varió significativamente en la prueba $S J$ en un $5.78 \pm 19.18 \%(\mathrm{p} \leq 0.05$ y 0.01$)$, CMJ en un $4.69 \pm 19.18 \%$ $(\mathrm{p} \leq 0.05$ y 0.01$)$ y CMJas $(\mathrm{p} \leq 0.05$ y 0.01$)$ en un $6.77 \pm 20.80 \%(\mathrm{p} \leq 0.05$ y 0.01 ) para el GE. En el caso del GC, los registros del tiempo de vuelo pre/ pos para SJ fueron de un $-2.65 \pm 29.53 \%$ CMJ, un $-19.84 \pm 15.07 \%$ y CMJas en un $-1.57 \pm 44.37 \%$ sin diferencias significativas encontradas.

En la Tabla 3 se muestran los registros para la velocidad de despegue (ms) en las pruebas de SJ, CMJ y CMJas con diferencias significativas ( $\mathrm{p} \leq$ 0.05 y 0.01 ) en favor del GE y con respecto al GC, tras 12 semanas de entrenamiento funcional. La velocidad de despegue $(\mathrm{ms})$ pre/pos varió significativamente en la prueba $\mathrm{SJ}$ en un $5.62 \pm 0.08 \%$ ( $\mathrm{p} \leq 0.05$ y 0.01 ), CMJ en un $4.74 \pm 0.08 \%(\mathrm{p} \leq 0.05$ y 0.01$)$ y CMJas en un $5.51 \pm 0.12 \%$ ( $\mathrm{p} \leq 0.05$ y 0.01$)$ para el GE. En el caso del GC, los registros de la velocidad de despegue (ms) pre/pos para SJ fueron de un $2.31 \pm 0.14 \%$, CMJ un $20.36 \pm 0.07 \%$ y CMJas $3.91 \pm 0.20 \%$, sin diferencias significativas encontradas. 
Tabla 3. Indicadores de desempeño para las pruebas de salto

\begin{tabular}{|c|c|c|c|c|c|c|c|}
\hline \multirow[t]{2}{*}{ Variable } & \multicolumn{2}{|c|}{ GE (n=12) } & \multicolumn{2}{|c|}{ GC (n=14) } & \multirow{2}{*}{$\begin{array}{c}\text { GE } \\
\text { Comparación } \\
\text { intramuestral }\end{array}$} & \multirow{2}{*}{$\begin{array}{c}\mathrm{GC} \\
\text { Comparación } \\
\text { intramuestral }\end{array}$} & \multirow{2}{*}{$\begin{array}{c}\text { GE VS GC } \\
\text { Comparación } \\
\text { intermuestral } \\
\text { (Post-test) }\end{array}$} \\
\hline & Pre-test & Post- test & Pre-test & Post- test & & & \\
\hline $\begin{array}{l}\text { Altura } \\
\text { SJ (cm) }\end{array}$ & $4.2 \pm 2.06$ & $27.28 \pm 2.25$ & $22.82 \pm 3.13$ & $24.03 \pm 3.35$ & $* *$ & $\mathbb{S}$ & $*$ \\
\hline $\begin{array}{c}\text { Altura } \\
\mathrm{CMJ}(\mathrm{cm})\end{array}$ & $25.14 \pm 2.32$ & $27.66 \pm 2.11$ & $24.59 \pm 1.66$ & $25.01 \pm 1.74$ & $* *$ & $\mathbb{S}$ & * \\
\hline $\begin{array}{l}\text { Altura CMJas } \\
\qquad(\mathrm{cm})\end{array}$ & $28.80 \pm 3.80$ & $33.04 \pm 3.64$ & $26.64 \pm 5.05$ & $27.43 \pm 4.97$ & $* *$ & $\$$ & * \\
\hline $\begin{array}{l}\text { Tiempo de } \\
\text { vuelo } \\
\text { SJ (ms) }\end{array}$ & $\begin{array}{c}443.41 \pm \\
18.28\end{array}$ & $\begin{array}{c}470.66 \pm \\
20.08\end{array}$ & $430 \pm 28.95$ & $\begin{array}{c}441.71 \pm \\
30.11\end{array}$ & $* *$ & $\$$ & * \\
\hline $\begin{array}{l}\text { Tiempo de } \\
\text { vuelo } \\
\text { CMJ (ms) }\end{array}$ & $\begin{array}{c}451.75 \pm \\
20.49\end{array}$ & $474 \pm 18.51$ & $360 \pm 14.20$ & $\begin{array}{c}449.14 \pm \\
15.95\end{array}$ & $* *$ & $\mathbb{S}$ & * \\
\hline $\begin{array}{l}\text { Tiempo de } \\
\text { vuelo CMJas } \\
(\mathrm{ms})\end{array}$ & $\begin{array}{c}482.75 \pm \\
30.13\end{array}$ & $\begin{array}{c}517.83 \pm \\
27.48\end{array}$ & $\begin{array}{c}463.21 \pm \\
42.03\end{array}$ & $\begin{array}{c}470.64 \pm \\
40.72\end{array}$ & $* *$ & $\mathbb{S}$ & * \\
\hline $\begin{array}{l}\text { Velocidad } \\
\mathrm{SJ}(\mathrm{cm} / \mathrm{ms})\end{array}$ & $2.18 \pm 0.08$ & $2.31 \pm 0.09$ & $2.11 \pm 0.14$ & $2.16 \pm 0.14$ & $* *$ & $\$$ & * \\
\hline $\begin{array}{c}\text { Velocidad } \\
\mathrm{CMJ}(\mathrm{cm} / \mathrm{ms})\end{array}$ & $2.21 \pm 0.09$ & $2.32 \pm 0.08$ & $1.76 \pm 0.68$ & $2.21 \pm 0.07$ & $* *$ & $\mathbb{S}$ & * \\
\hline $\begin{array}{l}\text { Velocidad } \\
\text { CMJas } \\
\text { (cm/ms) }\end{array}$ & $2.40 \pm 0.12$ & $2.54 \pm 0.13$ & $2.21 \pm 0.31$ & $2.30 \pm 0.19$ & $* *$ & $\mathbb{S}$ & $*$ \\
\hline
\end{tabular}

Nota: ** valor $\mathrm{p} \leq 0.001 ; \mathbb{\$}=$ No significativo; * valor $\mathrm{p} \leq 0.05$.

Fuente: elaboración propia.

No hubo diferencias significativas ( $\mathrm{p} \leq 0.05$ y 0.01 ) entre el GE y el GC para las pruebas de 200 y 50 metros estáticos, tras 12 semanas de entrenamiento funcional. Aunque no existen valores de mayor significancia, los tiempos promedios en el GE disminuyeron un $0.89 \pm 1.04 \%$ en la prueba de 200 metros estáticos y $3.11 \pm 0.44 \%$ en 50 metros estáticos. En tanto los valores para el GC permanecieron estables entre los momentos pre y pos, ya que los registros fueron de $0.01 \pm 19 \%$ para la prueba de 200 metros estáticos y de $0.82 \pm 0.41 \%$ para la prueba de 50 metros estáticos.

En la Tabla 4 se presentan los tiempos promedios para las distancias de 50, 100, 150 y 200 metros en las pruebas dinámicas, donde el GE con respecto al GC, presentó diferencias significativas ( $\mathrm{p} \leq 0.05$ y 0.01$)$ tras el 
programa de entrenamiento funcional y después de 12 semanas. En el caso del GE hubo una disminución significativa de $2.49 \pm 0.33 \%$ ( $\mathrm{p} \leq 0.05$ y 0.01 ) para el tramo de 50 metros, de $19.92 \pm 0.77 \%$ ( $\mathrm{p} \leq 0.05$ y 0.01 ) para el tramo de 100 metros, de $1.67 \pm 0.90 \%$ ( $\mathrm{p} \leq 0.05$ y 0.01$)$ para el tramo de 150 metros y de $0.77 \pm 1.1 \%$ ( $\leq \leq 0.05$ y 0.01 ) para el tramo de 200 metros. Por otro lado, el GC no aumentó significativamente los valores promedio por tramo ya que los registros fueron de $0.81 \pm 0.36 \%$ para el tramo de 50 metros, $0.54 \pm 0.68 \%$ para el tramo de 100 metros, $0.84 \pm 0.94 \%$ para el tramo de 150 metros y $1.01 \pm 1.24 \%$ para el tramo de 200 metros, lo cual refleja una menor capacidad para trasladar el cuerpo sobre los trayectos valorados y en el menor tiempo posible.

La velocidad, la aceleración, la fuerza y la potencia fueron estadísticamente diferentes ( $p \leq 0.05$ y 0.01 ) entre el GE y el GC para el tramo de 100 metros, con un cambio a favor del GE. En el caso del GE hubo un incremento de $16.74 \pm 0.51 \%$ ( $\mathrm{p} \leq 0.05$ y 0.01 ) en la velocidad, de $31.25 \pm 0.08$ $\%(\mathrm{p} \leq 0.05$ y 0.01$)$ en la aceleración, de $42.70 \pm 7.86 \%$ ( $\mathrm{p} \leq 0.05$ y 0.01 ) en la fuerza, y de $19.51 \pm 66.30 \%$ ( $\mathrm{p} \leq 0.05$ y 0.01 ) en la potencia; mientras que el GC solo aumenta un $0.44 \pm 0.56 \%$ en la velocidad, y en el caso de la aceleración los valores se incrementaron en $1.20 \pm 0.10 \%$, en $0.73 \pm 10.80 \%$ para la fuerza, y para la potencia un $1.14 \pm 15 \%$, cambios porcentuales que no fueron significativos. 
Tabla 3. Indicadores de capacidad condicional velocidad 200 metros para el GE y el GC después de la aplicación de un programa de entrenamiento funcional

\begin{tabular}{|c|c|c|c|c|c|c|c|}
\hline \multirow[t]{2}{*}{ Variable } & \multicolumn{2}{|c|}{$\mathrm{GE}(\mathrm{n}=12)$} & \multicolumn{2}{|c|}{ GC $(n=14)$} & \multirow{2}{*}{$\begin{array}{c}\text { GE } \\
\text { Comparación } \\
\text { Intramuestral }\end{array}$} & \multirow{2}{*}{$\begin{array}{c}\text { GC } \\
\text { Comparación } \\
\text { Intramuestral }\end{array}$} & \multirow{2}{*}{$\begin{array}{c}\text { GE VS GC } \\
\text { Comparación } \\
\text { intermuestral } \\
\text { (Post-test) }\end{array}$} \\
\hline & Pre-test & Post- test & Pre-test & Post- test & & & \\
\hline Tiempo (ms)50 m & $6.17 \pm 0.31$ & $6.02 \pm 0.36$ & $6.16 \pm 0.39$ & $6.11 \pm 0.34$ & $\$$ & $\$$ & $\mathbb{S}$ \\
\hline $\begin{array}{c}\text { Tiempo (ms)100 } \\
\text { m }\end{array}$ & $13.54 \pm 0.68$ & $\begin{array}{c}11.29 \pm \\
0.86\end{array}$ & $11.09 \pm 0.71$ & $11.03 \pm 0.66$ & $n *$ & $\$$ & $*$ \\
\hline $\begin{array}{c}\text { Tiempo }(\mathrm{ms}) 150 \\
\mathrm{~m}\end{array}$ & $17.03 \pm 0.90$ & $\begin{array}{c}16.75 \pm \\
0.91\end{array}$ & $16.76 \pm 0.93$ & $16.62 \pm 0.96$ & $\mathbb{S}$ & $\$$ & $\$$ \\
\hline $\begin{array}{c}\text { Tiempo (ms)200 } \\
\text { m }\end{array}$ & $22.08 \pm 1.06$ & $\begin{array}{c}21.91 \pm \\
1.14\end{array}$ & $21.82 \pm 1.24$ & $21.60 \pm 1.25$ & $\mathbb{S}$ & $\$$ & $\mathbb{S}$ \\
\hline $\begin{array}{l}\text { Velocidad } \\
(\mathrm{m} / \mathrm{ms}) 50 \mathrm{~m}\end{array}$ & $8.12 \pm 0.42$ & $8.33 \pm 0.53$ & $8.14 \pm 0.51$ & $8.20 \pm 0.46$ & $\mathbb{S}$ & $\$$ & $\mathbb{S}$ \\
\hline $\begin{array}{l}\text { Velocidad } \\
(\mathrm{m} / \mathrm{ms}) 100 \mathrm{~m}\end{array}$ & $7.41 \pm 0.39$ & $8.90 \pm 0.63$ & $9.05 \pm 0.57$ & $9.09 \pm 0.55$ & w* & $\$$ & $*$ \\
\hline $\begin{array}{l}\text { Velocidad } \\
(\mathrm{m} / \mathrm{ms}) 150 \mathrm{~m}\end{array}$ & $8.83 \pm 0.47$ & $8.98 \pm 0.50$ & $8.97 \pm 0.51$ & $9.05 \pm 0.52$ & $\mathbb{S}$ & $\$$ & $\$$ \\
\hline $\begin{array}{l}\text { Velocidad } \\
(\mathrm{m} / \mathrm{ms}) 200 \mathrm{~m}\end{array}$ & $9.08 \pm 0.45$ & $9.15 \pm 0.49$ & $9.19 \pm 0.53$ & $9.28 \pm 0.54$ & $w *$ & $\$$ & $*$ \\
\hline $\begin{array}{l}\text { Aceleración } \\
\left(\mathrm{m} / \mathrm{ms}^{2}\right) 50 \mathrm{~m}\end{array}$ & $1.32 \pm 0.14$ & $1.39 \pm 0.18$ & $1.33 \pm 0.17$ & $1.35 \pm 0.15$ & $\$$ & $\$$ & S \\
\hline $\begin{array}{l}\text { Aceleración } \\
\left(\mathrm{m} / \mathrm{ms}^{2}\right) 100 \mathrm{~m}\end{array}$ & $0.55 \pm 0.06$ & $0.80 \pm 0.11$ & $0.82 \pm 0.10$ & $0.83 \pm 0.10$ & $n *$ & $\$$ & $*$ \\
\hline $\begin{array}{l}\text { Aceleración } \\
\left(\mathrm{m} / \mathrm{ms}^{2}\right) 150 \mathrm{~m}\end{array}$ & $0.52 \pm 0.06$ & $0.54 \pm 0.06$ & $0.54 \pm 0.06$ & $0.55 \pm 0.06$ & $\mathbb{S}$ & $\$$ & $\mathbb{S}$ \\
\hline $\begin{array}{l}\text { Aceleración } \\
\left(\mathrm{m} / \mathrm{ms}^{2}\right) 200 \mathrm{~m}\end{array}$ & $0.41 \pm 0.04$ & $0.42 \pm 0.05$ & $0.42 \pm 0.05$ & $0.43 \pm 0.05$ & $\$$ & $\$$ & $\mathbb{S}$ \\
\hline $\begin{array}{c}\text { Fuerza } \\
\left(\mathrm{kg}^{*} \mathrm{~m} / \mathrm{ms}^{2}\right) 50 \mathrm{~m}\end{array}$ & $26.38 \pm 8.27$ & $\begin{array}{l}25.33 \\
\pm 3.82\end{array}$ & $26.52 \pm 5.24$ & $26.55 \pm 5.35$ & $\mathbb{S}$ & $\$$ & $\$$ \\
\hline $\begin{array}{c}\text { Fuerza } \\
\left(\mathrm{kg}^{*} \mathrm{~m} / \mathrm{ms}^{2}\right) 100 \mathrm{~m}\end{array}$ & $63.66 \pm 7.29$ & $\begin{array}{c}44.61 \pm \\
8.44\end{array}$ & $43.30 \pm 10.61$ & $\begin{array}{c}43.62 \pm \\
11.00\end{array}$ & $* *$ & $\$$ & * \\
\hline $\begin{array}{c}\text { Fuerza } \\
\left(\mathrm{kg}^{*} \mathrm{~m} / \mathrm{ms}^{2}\right) 150 \mathrm{~m}\end{array}$ & $67.04 \pm 6.30$ & $\begin{array}{l}65.10 \pm \\
7.26\end{array}$ & $65.50 \pm 13.10$ & $\begin{array}{c}65.36 \pm \\
13.16\end{array}$ & $\$$ & $\$$ & $\mathbb{S}$ \\
\hline $\begin{array}{c}\text { Fuerza } \\
\left(\mathrm{kg}^{*} \mathrm{~m} / \mathrm{ms}^{2}\right) 200 \mathrm{~m}\end{array}$ & $84.57 \pm 7.93$ & $\begin{array}{l}83.44 \pm \\
8.27\end{array}$ & $82.88 \pm 14.61$ & $\begin{array}{c}82.68 \pm \\
15.70\end{array}$ & $\mathbb{S}$ & $\$$ & $\mathbb{S}$ \\
\hline $\begin{array}{l}\text { Potencia } \\
(\mathrm{J} / \mathrm{s}) 50 \mathrm{~m}\end{array}$ & $\begin{array}{c}214.50 \pm \\
22.91\end{array}$ & $\begin{array}{c}210.27 \pm \\
28.96\end{array}$ & $215.55 \pm 41.49$ & $\begin{array}{c}217.42 \pm \\
42.38\end{array}$ & $\mathbb{S}$ & $\$$ & $\mathbb{S}$ \\
\hline $\begin{array}{l}\text { Potencia } \\
(\mathrm{J} / \mathrm{s}) 100 \mathrm{~m}\end{array}$ & $\begin{array}{c}471.17 \pm \\
57.55\end{array}$ & $\begin{array}{l}394.24 \\
\pm 55.80\end{array}$ & $389.50 \pm 85.11$ & $\begin{array}{c}394.00 \pm \\
87.30\end{array}$ & $* *$ & $\$$ & * \\
\hline $\begin{array}{l}\text { Potencia } \\
(\mathrm{J} / \mathrm{s}) 150 \mathrm{~m}\end{array}$ & $\begin{array}{l}591.82 \pm \\
66.74\end{array}$ & $\begin{array}{l}582.10 \pm \\
66.85\end{array}$ & $\begin{array}{c}586.63 \pm \\
115.23\end{array}$ & $\begin{array}{c}589.50 \pm \\
116.15\end{array}$ & $\mathbb{S}$ & $\$$ & $\mathbb{S}$ \\
\hline $\begin{array}{l}\text { Potencia } \\
(\mathrm{J} / \mathrm{s}) 200 \mathrm{~m}\end{array}$ & $\begin{array}{l}768.06 \pm \\
86.92\end{array}$ & $\begin{array}{l}763.67 \pm \\
86.76\end{array}$ & $762.34 \pm 140.33$ & $\begin{array}{l}67.25 \pm \\
145.31\end{array}$ & $\mathbb{S}$ & $\$$ & $\$$ \\
\hline
\end{tabular}

Nota: ${ }^{* *}=$ valor $\mathrm{p} \leq 0.001 ; \mathbb{\$}=$ No significativo; * valor $\mathrm{p} \leq 0.05$.

Fuente: elaboración propia. 


\section{Variables antropométricas}

Tras 12 semanas de entrenamiento funcional, hubo ligeras variaciones entre el GE y el GC, con cambios significativos en el perímetro de la pierna. Estos resultados coinciden con los descrito por Lloyd y Oliver (2012) y Pääsuke, Ereline y Gapeyeva (2001) en relación a las alteraciones del perfil morfológico y funcional de niños. Los resultados describen una ganancia en las capacidades condicionales (p. ej. fuerza y velocidad), sin alteración de los compartimientos corporales, lo cual puede ser explicado por una mejora de la actividad del sistema nervioso y su influencia sobre los patrones de coordinación agonista y antagonista que han sido referenciados en diferentes trabajos.

Los resultados del presente estudio son ligeramente diferentes a lo informado por Lloyd y Oliver (2012) y Pääsuke et al. (2001), los cuales describen diferencias en el peso que pueden ser explicadas desde las variables del entrenamiento, ya que los ciclos empleados no coinciden plenamente entre los trabajos. No obstante, nuevamente se reporta la modificación significativa del perímetro del muslo entre niños y niñas antes de la pubertad, lo cual advierte de una mejora de las expresiones de fuerza que puede estar relacionada con la modificación del área de sección transversal de la sección del muslo (Hakkarainen et al., 2009).

\section{Variables de fuerza explosiva frente a la independencia funcional}

Teniendo en cuenta los resultados de las variables relacionadas con la fuerza explosiva, se destaca que hubo una mejora de la expresión funcional de miembros inferiores de los infantes intervenidos tras 12 semanas de entrenamiento de fuerza basados en un programa funcional. Dichos cambios podrían estar relacionados con adaptaciones neurales como, por ejemplo, cambios en los patrones de coordinación, componente muscular, donde se aprecian actividades diferenciadas del tipo de fibras musculares e incremento del área de sección transversal muscular, así como alteraciones osteoarticulares conectadas a la morfología de las estructuras y al fortalecimiento del tejido blando (ligamentos y tendones). 
Por otro lado, Alegre (2004) ha descrito una correlación de 0.57 $(\rho<0.05)$ entre los cambios en la altura en SJ y los cambios en 1 RM en $1 / 2$ salto desde media sentadilla, tras un programa de entrenamiento de 13 semanas, realizando 3 sesiones por semana, en sujetos físicamente activos. Sin embargo, los saltos tienen tendencia a entrenar más las propiedades elásticas de cuádriceps y glúteos, aumentando la potencia de los mismos, ya que estos músculos por la posición corporal asumida durante la práctica del patinaje tienen mayor incidencia en el paso del patinador durante la curva y la recta (Lugea, 2010); por consiguiente, se puede establecer que la tendencia a mejorar las expresiones de tensión en ciclo de estiramiento y acortamiento en una sesión de entrenamiento, se puede generar mediante lo que se conoce como transferencia a los gestos técnicos, en este caso con saltos imitativos de la disciplina deportiva patinaje de velocidad.

Sobre el tema, aunque no se deben descartar algunos estudios donde se describe la posibilidad de hipertrofia en niños (Behm, Faigenbaum, Falk y Klentrou, 2008), en general existe consenso en que los cambios alcanzados mediados por el entrenamiento en la infancia están fuertemente asociados a la mejora en los patrones de coordinación, más que a fenómenos hipertróficos o hiperplásicos (Ozmun et al., 1994; Ramsay et al., 1990).

\section{Variables funcionales test 200 metros salida estática}

Se resalta para la prueba de 200 metros que al disminuir los tiempos en las pruebas de 200 y 50 metros, la velocidad aumenta para el GE en aproximadamente un $1 \%(0.86 \pm 0.36 \%)$, comparado con el GC. Al respecto, un aspecto que debe ser considerado para la eficiencia en la técnica de salida para patinadores podría ser el método asistido empleado en prueba de la velocidad estática, como lo sugiere Publow (1999).

Sobre el tema se puede advertir que el entrenamiento funcional, donde se incluyeron ejercicios unilaterales, bipodales y horizontales de todo el cuerpo, tuvo efectos positivos y permitió hallar ganancias significativas en la aceleración, potencia, fuerza y velocidad en el tramo de los 100 metros de la prueba de 200 metros. Dichos aspectos pueden estar ligados a adaptaciones neurales (mayor coordinación intramuscular e intermuscular) y podrían 
ser medidos de manera indirecta por el tiempo empleado para transferir las ganancias de tensión a las habilidades deportivas (Young y Willey, 2009).

\section{Conclusiones}

Con relación al entrenamiento en edades tempranas, es valioso recalcar la importancia de centrar los procesos en el desarrollo de las habilidades motrices, más que en capacidades condicionales como advierte Meinel (2004), donde modelos basados en la estimulación funcional como el empleado en el presente estudio, permiten no solo cumplir con el objetivo sugerido, sino también con la inclusión de actividades variadas y jugadas que pueden tener un efecto significativo y positivo en la funcionalidad del deportista de patinaje, aspecto que las proyecta como una alternativa a los modelos de trabajo convencional propios de la disciplina deportiva.

Si bien los resultados presentados son interesantes y valiosos para los procesos de formación infantil, se sugiere para estudios futuros incrementar el tamaño de los grupos, extender la duración total del programa y combinar el modelo con ligeras sobrecargas, lo cual podría potenciar los efectos positivos sobre la capacidad funcional general de los niños atletas.

A modo de conclusión, un programa de entrenamiento funcional como el del presente estudio, centrado en la fuerza muscular y aplicado en patinadores en formación con edades de entre 9 y 11 años, induce efectos positivos sobre la capacidad de velocidad de desplazamiento en diferentes tramos sin alterar significativamente las características morfológicas del deportista. Además, dichas ganancias son obtenidas en un corto período de tiempo y permiten incluir actividades lúdicas y recreativas con el propio peso corporal, lo cual favorece los procesos formativos en edades tempranas.

\section{Referencias}

Alegre, L. M. (2004). Cambios en la arquitectura y biomecánica del músculo esquelético tras un entrenamiento de fuerza explosiva. Universidad de Castilla la Mancha, Toledo, España. Recuperado de https://previa.uclm.es/profesorado/ xaguado/ASIGNATURAS/tesis/TesisLuisMariaAlegre.pdf 
American Academy of Pediatrics. (2000). Intensive training and sports specialization in young athletes. American Academy of Pediatrics. Committee on Sports Medicine and Fitness. Pediatrics, 106(1), 154-7. DOI: https://doi.org/10.1542/ peds.106.1.154

American College of Sports Medicine. (1993). The Prevention of Sports Injuries of Children and Adolescents. Medicine \& Science in Sports \& Exercise, 25(8), 1-7. https://doi.org/10.1249/00005768-199308001-00001

Aón, B. (2011). Introducción al entrenamiento funcional. Curso Posgrado Entrenamiento Funcional.

Aón, J., Birrum, R. y Lafranco, M. (2001). Introducción al entrenamiento funcional. Curso Protocolos Funcionales.

Behm, D., Faigenbaum, A., Falk, B. y Klentrou, P. (2008). Canadian Society for Exercise Physiology position paper: resistance training in children and adolescents. Applied Physiology, Nutrition, and Metabolism, 33, 547-61. DOI: https://doi.org/10.1139/h08-020

Bompa, T. (2003). Periodización. Teoría y metodología del entrenamiento. Barcelona, España: Editorial Hispano Europea.

Bosco, C. (1994). La valoración de la fuerza con el test de Bosco. Barcelona, España: Paidotribo.

Blimkie, C., Ramsay, J., Sale, D., MacDougall, D., Smith, K. y Garner, S. (1989). Effects of 10 weeks of resistance training on strength development in prepubertal boys. En Oseid y Carlsen (eds.), Children and Exercise, XIII, 183-197. Human Kinetics Champaing.

Chulvi, C. (2008). Criterios para la planificación y el desarrollo de programas de acondicionamiento muscular en el ámbito de la salud. En Rodríguez P., Ejercicio físico en salas de acondicionamiento muscular (pp. 91-127). Madrid, España: Panamericana.

Faigenbaum, A., Zaichkowsky, L., Westcott, W., Micheli, L. \& Fehlandt, A. (1993). The Effects of Twice a Week Strength Training Program on Children. Pediatric Exercise Science, 5, 339-346. DOI: https://doi.org/10.1123/pes.5.4.339.

Heredia, J. y Peña, G. (2011). Criterios para el diseño de programas de entrenamiento funcional. Instituto Universitario de Educación Física, Universidad de Antioquia, Medellín, Colombia.

Heredia, E. Chulvi, I., Donate, F., Soro, J. y Costa, M. (2007). Determinación de la carga de entrenamiento para la mejora de la fuerza orientada a la salud (fitness 
muscular). EF Deportes [en linea], 1-24. DOI: https://doi.org/10.19083/ tesis/624818

Hakkarainen, H., Jaakkola, T., Kalaja, S., Lämsä, J., Nikander, Y. A. \& Riski, J. (2009). Lasten Ja Nuorten Urheiluvalmennuksen Perusteet, 1. Painos, Jyväskylä, VK-Kustannus

Lugea, C. (2010). Posiciones y saltos de potenciación para el patinaje de velocidad, versión 1.7-EMDER/AMP. Recuperado de http://ipatinantioquia.com.co/ imagenes/originalpdf/9262Posicionesysaltosparaelpatinaje1.7.pdf

Lloyd, R. y Oliver, J. (2012). El modelo de desarrollo físico de la juventud: una nueva aproximación a largo plazo atlético desarrollo, fuerza y acondicionamiento diario. $10^{\circ}$ Seminario Internacional de Entrenamiento Deportivo. 34(3), 61-72. Recuperado de https://1968.com.ve/wp-content/uploads/2016/01/ Posicionamiento-sobre-el-entrenamiento-de-fuerza-en-jovenes.pdf

Marfell-Jones, M. (2001). Manual ISAK: estándares de medidas antropométricas internacionales. Durban, Suráfrica: Sociedad Internacional para el Avance de la Cineantropometría. Recuperado de https://scholar.google.es/scholar?cluster $=13841690656260842676 \& \mathrm{hl}=$ es\&as_sdt $=2005 \&$ sciodt $=0,5$

Meinel, K. (2004). Teoría del movimiento. 2da edición. Buenos Aires, Argentina: Stadium.

Naclerio, F. (2011). Curso a distancia de entrenamiento en deportes acíclicos (primera edición). Modulo 1, Metodología del entrenamiento de la potencia muscular en deportes acíclicos. DOI: https://doi.org/10.21892/01239813.166

Ozmun, J., Mikesky, A. y Surburg, P. (1994). Neuromuscular adaptations following pre-pubescent strength training. Medicine \& Science in Sports \& Exercise, 26(4), 510-514. DOI: https://doi.org/10.1249/00005768-199404000-00017

Pääsuke, M., Ereline, J. y Gapeyeva, H. (2001). Fuerza rodilla extensor muscular y características de rendimiento de salto vertical en pre y post-puberales niños, Pediátrica, Ciencias del Ejercicio, 13, 60-69. DOI: https://doi.org/10.1123/ pes.13.1.60

Publow, B. (1999). Velocidad en patines, una técnica, capacitación y guía completa de carreras en línea y los patinadores de hielo. Windsor, Reino Unido: Human Kinetics.

Platonov, V. (2001). Teoría general del entrenamiento deportivo olímpico. Barcelona, España: Editorial Paidotribo.

Quintana, M. (2005). Teoría de la cineantropometría. Madrid, España: INEF. 
Ramsay, J., Blimkie, C., Smith, K., Garner, S., McDougall, J., Sale, D. (1990). Strength training effects in prepubescent boys. Medicine \& Science in Sports or Exercise, 22(5), 605-614. DOI: https://doi. org/10.1249/00005768-199010000-00011

Ravé, J. Valdivielso, F. y Gaspar, P. (2007). La planificación del entrenamiento deportivo: cambios vinculados a las nuevas formas de entender las estructuras deportivas contemporáneas. Conexões, 5(1),1-22.DOI: https://doi.org/10.20396/ conex.v5i1.8637976.

Romero, S. Feria, A., Sañudo, B., De Hoyo M. y Del Ojo, J. (2014). Efectos de entrenamiento de fuerza en sistema isoinercial sobre la mejora del CMJ en jóvenes futbolistas de elite. Retos. Nuevas tendencias en educación física, deporte y recreación, (26). DOI: https://doi.org/10.24310/riccafd.2013.v2i3.6194

Sailors, M. y Berg, K. (1987). Comparison of responses to weight training in pubescent boys and men. The Journal of sports medicine and physical fitness, 27(1), 30-37.

Young, W. y Willey, B. (2009). Analysis of a reactive agility field test. Journal of science and medicine in sport / Sports Medicine Australia, 13(3), 376-8. DOI: https://doi.org/10.1016/j.jsams.2009.05.006. 